\title{
Air-Dried and wet fixation on Fine Needle Aspiration Biopsy (FNAB) specimen
}

\author{
Fitri Nuroini $\square$, Zaldy Alfanda Hidayat $\square$, Tulus Ariyadi $\square$ \\ Medical Laboratory Technology, Nursing and Health Faculty, Universitas Muhammadiyah \\ Semarang, Indonesia
}

\begin{abstract}
Two fixation methods can affect the quality of cytological staining derived from fine-needle aspiration biopsy, but both fixation methods have drawbacks. Dry fixation can cause cell rupture, while wet fixation requires a longer time and costs more, so dry fixation is more often used. The purpose of this study was to compare the results of the Diff-Quick staining of cytological preparations that were fixed with the dry fixation method and the wet fixation method on FNAB samples. This study was an experimental study with 36 samples of cytology preparations from FNAB, divided into three groups, the control group, dry fixation group, and wet fixation group. Staining dry fixation preparations gave four poor results, five good preparations, and three very good preparations while staining wet fixation preparations gave 0 poor results, eight good preparations, and four very good preparations. This shows that the staining results of wet-fixed preparations tend to be of better quality and more consistent than the staining results of dry-fixed preparations. Even though statistically through the test Post-Hoc showed no difference, the quality of Diff-Quick staining of cytological preparations fixed by wet fixation method was better than dry fixation, so the wet fixation method was more recommended for cytological preparations than FNAB.
\end{abstract}

Keywords: Air-dried fixation; Wet fixation; FNAB; Diff-Quick.

\section{INTRODUCTION}

Cytology specimens are easy to obtain in the laboratory, but proper technique in collecting cytological specimens needs to be considered because it can affect the examination results..$^{-}$Cytological specimen collection techniques are divided based on the type of specimens such as rinses in the stomach, bladder, and bronchi, or scraping as in the Pap Smear, as well as those collected directly such as sputum and urine. ? In addition, specimens can also be obtained from FNAB ( Fine Needle Aspiration Biopsy), which is widely used and is a quick and inexpensive diagnostic technique. ${ }^{3}$ The FNAB technique can be used to diagnose breast cancer, namely to determine the presence of cell malignancies. 4 In addition, evaluation of samples from FNAB is vital for the pathologist to confirm sample adequacy, additional tests and establish an initial cytologic interpretation for the tumor. $1, \underline{5}$

Specimens from FNAB are usually received directly in the form of smears taken by medical personnel who have met the requirements or have received training. 6,7 The smear from the FNAB then will be fixed and stained with the

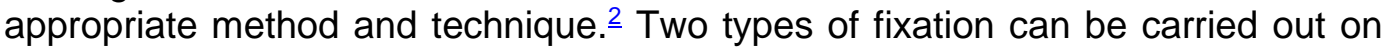
cytological specimens in the form of smears, namely wet fixation and dry fixation. ${ }^{7}$ Wet fixation using alcohol and stained with Papanicolaou or 
Hematoxylin Eosin. While dry fixation using Romanonowsky staining., 9 . Wet fixation with Papanicolaou stain is recommended for staining core morphology for specimens from FNAB and effusions, while dry fixation is recommended for viewing details of the cytoplasm. ${ }^{7}$

Fixation on cytological smear preparations can affect the condition and stability of cells, prevent loss of cell content, display reactive sites for staining, and increase cell membrane permeability for dyes. 10 Dry fixation and wet fixation methods are commonly used in the fixation of cytological preparations. The wet fixation method can keep the cell conditions as close as possible to the conditions when they were still in the body. $.11,12$ Fixation solutions used in the wet method include alcohol, methanol, propanol, isopropanol, and formalin. The dry fixation method can be done by drying the preparation in the open air or heating by hairdryer. $\frac{13}{2}$ The dry fixation method is relatively more manageable, cheaper, and shorter in operation than the wet fixation method and can prevent the occurrence of artifacts. However, dry fixation can result in an increase in cell size, an apparent effect on the size and shape of the cell nucleus., $, 8,14$ The speed with which the results of the cytological examination are released is essential. Therefore the fixation is not too long to reduce the waiting time required of the patient to obtain the results. $\frac{10,12}{}$ Health agencies will usually choose a fixation method according to the needs and the available reagents and tools. The choice of fixation method for cytology preparations is adjusted to the quality of the preparation, time, and cost-efficiency.

The fixation of cytological preparations is one of the factors that can affect the staining of the preparations. Papanicolaou stain is commonly used for cytological samples but has the disadvantage, its takes a long time in the staining process..$\frac{15}{}$ One of the commonly used Romanowsky stains because it is easy to do, inexpensive, and does not require a long time so that the test results can be issued quickly is the Diff-Quick stain. $\underline{16}, \underline{17}$ Research that compares the results of Diff-Quick staining on cytological preparations from FNAB with the wet fixation method and the dry method needs to be done to determine which fixation method is better to use. Similar research has never been done before; therefore, this research needs to be done. This research will reference the use of a good and efficient method of fixation of cytological preparations from FNAB samples, both in terms of time and in terms of costs.

\section{MATERIAL AND METHOD}

This type of research is an experimental design with Post-test Only Group Design. The research was carried out in August 2020 at the Anatomical Pathology Laboratory at one of the General Hospitals in Semarang. The samples used were cytology preparations of patients in the Laboratory of Pathology and anatomy obtained through the FNAB method as 36 preparations. The preparations obtained were divided into three groups based on the fixation method used, namely the dry fixation method, wet fixation method, and control as a comparison. The dry fixation method was carried out by drying the preparation using a hairdryer until the preparation was dry. The wet fixation method is done by immersing the preparation in $95 \%$ alcohol solution for 1 minute. The control fixation group is the fixation used in the Pathology anatomic Laboratory, where the research was conducted by combining dry fixation and wet fixation. After all, preparations have been fixed, proceed with staining the preparations using the Diff-Quick stain, which is available in a Staining Kit. The staining procedure begins with each preparation dipping in 8 dips of Diff-Quick I (Eosin) solution, then dried. Then it was dipped in 8 diff-Quick II (Methylene blue) solution. The preparation is then rinsed in running water, then allowed to dry. An Anatomical Pathologist Specialist read the dried preparations. The criteria for reading the preparations are following Table 1. 
Table 1. Criteria for Assessment of Preparation Quality

\begin{tabular}{llll}
\hline \multirow{2}{*}{ Criteria } & \multicolumn{3}{c}{ Category } \\
\cline { 2 - 4 } & Not good & Well & Very good \\
\hline Cell shape & Unclear & Clear & Very clear \\
Nucleus & A colored core is & The core is & The colored core is \\
& not clear & colored & very clear \\
Nucleolus (child nucleus) & Not visible & Seen & See clearly \\
Cytoplasm and intracellular & Unclear & Clear & Very clear \\
components & & & \\
\hline
\end{tabular}

\section{RESULTS AND DISCUSSION}

The results of the microscopic study showed that the staining quality of the preparations fixed by the dry fixation method had less than optimal results. Poor staining results were only found in the preparations fixed by the dry fixation method, while similar results were not found in the preparations using the wet fixation method and controls (table 2). Several factors that can cause this to happen include inadequate drying so that there is still water in the preparation. The presence of water content in the preparation is a phenomenon known as a drying artifact. 18 This phenomenon can be observed in the picture of red blood cells that are round or sickle, with cells that look like holes or have a structure like a refractile vacuole in Figure 2. In addition, the presence of water content in the smear preparation will cause the cells to become moist, which will eventually result in the dye cannot bind perfectly to the cell components in the smear preparation. The result is that the preparation is not appropriately stained, such as the results of research by Jhala that dry fixation is a fixation method that is often used in diff-quick staining, but if the drying process is not suitable, it can cause poor staining results and detail pictures. $\frac{19}{}$

The results also showed that the microscopic appearance of cells for the preparations fixed by the dry fixation method was less than optimal. In addition, cell details such as the nucleus and nucleolus are less observable. The slow drying process can cause the cell to become hypertonic due to the evaporation of water that occurs by increasing the temperature, which causes the rupture of the cell membrane and nuclear membrane. $\frac{10}{10}$ This is also in line with the study results that the preparations fixed by the dry fixation method had relatively fewer cells that could be observed compared to the preparations fixed by the wet method or in the control preparations. Factors allow this to occur due to cell rupture due to drying using a hairdryer. $\frac{18}{}$

Preparations fixed with alcohol can cause the blood smear to be insoluble in water, whereas this is not found in preparations fixed by drying. This occurs because of the effect of the alcohol fixation solution, which reduces the electric charge, and the decrease in the dielectric constant, which allows for stronger attractive bonds between protein molecules. $\underline{20}$ This is also one of the factors causing poor results in the preparations fixed by the dry fixation method because the cells can also dissolve with water. After all, the final process of diff-quick staining is rinsing the preparation with running water.

Good and very good staining results were obtained in all fixation methods because the three fixation methods can trigger the denaturation process. The denaturation process occurs due to the presence of solvents such as alcohol and also high temperatures. This condition causes an increase in density and a decrease in cell elasticity, and an increase in reactivity to acid-base dyes such as diff-quick dyes. The reaction is further because it involves the opening of globular proteins that increase the number of reactive groups capable of binding to acidbase dyes. $\underline{20}$ According to the research results, the dye can bind well to the cells so that the preparation is stained well or even very well. 
The results of the Kruskal-Wallis statistical test ( $p$-value $>0.05)$ showed that the quality of the staining results of the preparations fixed by the dry method and the wet method had no difference. The results of Post Hoc analysis between the staining results of wet-fixed and control preparations ( $p$-value $>0.05$ ) and between dry fixation and control ( $p$-value $<0.05)$, it can be interpreted that the preparations fixed by the wet method have similar staining results with statistical control when compared with the staining results of the preparations that were fixed by the dry method. However, microscopically, the staining results of the preparations fixed by the dry method did not show the same results as wet fixation. In addition, the results of staining with preparations fixed by the dry method were also inconsistent, as evidenced by the results obtained that were too varied compared to the wet fixation method, which did not show poor results and was consistent with good staining results. The results obtained in this study are in accordance with the research results conducted by Rupinder that wet fixation gave better results than a dry fixation on cervical smear samples. $\frac{11}{1}$ The same results were also shown by Randal \& van Amerongen 21 and the results of Safnect ${ }^{22}$. Different results were shown by Shiddam ${ }^{3}$ on the FNAB and Gupta samples $\underline{7}$ on cervical smear samples, dry fixation showed better staining results compared to wet fixation.

\section{CONCLUSION}

The research found that the staining quality of the cytological sample preparation from FNAB on diff-quick staining with wet fixation was better than dry fixation. According to the results of this study, wet fixation was preferred for cytological samples from FNAB compared to dry fixation. The results also showed that combining the two fixation methods as the control group in this study showed the best results for the FNAB sample.

\section{AUTHORS' CONTRIBUTIONS}

All authors contributed equally to this work.

\section{FOUNDING INFORMATION}

The funding for this research was sourced from University of Muhammadiyah Semarang.

\section{DATA AVAILABILITY STATEMENT}

The utilized data to contribute to this investigation are available from the corresponding author on reasonable request.

\section{DISCLOSURE STATEMENT}

The views and opinions expressed in this article are those of the authors and do not necessarily reflect the official policy or position of any affiliated agency of the authors. The data is the result of the author's research and has never been published in other journals.

\section{REFERENCE}

1. Choi US da. S, Kim DY. Immunocytochemical detection of Ki-67 in DiffQuik-stained cytological smears of canine mammary gland tumours. Cytopathology. 2011;22(2):115-120. doi:10.1111/i.13652303.2010.00756.x.

2. Al-Abbadi MA. Basics of cytology. Avicenna J Med. 2011;01(01):18-28. doi:10.4103/2231-0770.83719.

3. Shidham VB, Kampalath B, England J. Routine Air Drying of All Smears Prepared During Fine Needle Aspiration and Intraoperative Cytology Studies. Acta Cytol. 2001;45(1):60-68. doi:10.1159/000327188.

4. Khan MZ, Haleem A, Al Hassani H, Kfoury H. Cytopathological grading, as 

a predictor of histopathological grade, in ductal carcinoma (NOS) of breast, on air-dried Diff-Quik smears. Diagn Cytopathol. 2003;29(4):185-193. doi:10.1002/dc.10285.

5. Alsharif M, Carlo-Demovich J, Massey C, et al. Telecytopathology for immediate evaluation of fine-needle aspiration specimens. Cancer Cytopathol. 2010;118(3):119-126. doi:10.1002/cncy.20074.

6. Orell SR, Sterrett GF, Whitaker D. Fine Needle Aspiration Cytology. 5th ed. Elsevier Churchill Livingstone; 2005.

7. Gupta S. Rehydration of air-dried cervical smears: a feasible alternative to conventional wet fixation. Obstet Gynecol. 2003;102(4):761-764. doi:10.1016/S0029-7844(03)00771-3.

8. Krafts K, Pambuccian S. Romanowsky staining in cytopathology: history, advantages and limitations. Biotech Histochem. 2011;86(2):82-93. doi:10.3109/10520295.2010.515492.

9. Kapse SS, Arakeri SU, Yerranguntla DP. Rehydration of Air-Dried Smears with Normal Saline: An Alternative for Conventional Wet Fixation Method in Cervical Cytological Study. J Cytol. 2018;35(4):199-203. doi:10.4103/JOC.JOC 18617.

10. Jörundsson $\mathrm{E}$, Lumsden $\mathrm{JH}$, Jacobs RM. Rapid Staining Techniques in Cytopathology: A Review and Comparison of Modified Protocols for Hematoxylin and Eosin, Papanicolaou and Romanowsky Stains. Vet Clin Pathol. 1999;28(3):100-108. doi:10.1111/j.1939-165X.1999.tb01057.x.

11. Rupinder K, Shubra W, Kanwal M. Rehydration of Air-Dried Smears versus Wet Fixation: A Cross-Sectional Study. Acta Cytol. 2013;57(4):364368. doi:10.1159/000351362.

12. Lowe J, Anderson P, Stevens A. Lowe's Human Histology. 4th ed. Elsevier/Mosby; 2015.

13. Khristian E, Inderiati D. Sitohistoteknologi. Kementrian Kesehatan Republik Indonesia; 2017.

14. Narayanan O. DN, Bai DA. Comparison of different methods of fixation in Papanicolaou staining of cervical smears: Wet-fixation and rehydration of air dried smears. Trop J Pathol Microbiol. 2019;5(1):15-19. doi:10.17511/jopm.2019.i01.03.

15. Prasaad PR. Short-duration Papanicolaou stain (SPS) - an alternative to conventional Papanicolaou stain in routine cytopathology? Comp Clin Path. 2017;26(6):1285-1288. doi:10.1007/s00580-017-2524-1.

16. Jackson DE, Selting KA, Spoor MS, Henry CJ, Wiedmeyer CE. Evaluation of fixation time using Diff-Quik for staining of canine mast cell tumor aspirates. Vet Clin Pathol. 2013;42(1):99-102. doi:10.1111/vcp.12012.

17. Youssef D, Shams W, Ganote CE, Al-Abbadi MA. Negative Image of Blastomyces on Diff-Quik Stain. Acta Cytol. 2011;55(4):377-381. doi:10.1159/000327721.

18. Rick L Cowell, Meinkoth JH, Tayler RD, DeNicola DD. Diagnostic Cytology and Hematology of the Dog and Cat. 4 ed. St. Elsevier Inc; 2008.

19. Jhala N, Jhala D. Definitions in Tissue Acquisition. Gastrointest Endosc Clin N Am. 2014;24(1):19-27. doi:10.1016/i.giec.2013.08.005.

20. Wolman M. Problems of Fixation in Cytology, Histology, and Histochemistry. In: Histology. and Histochemistry. Internaitonal Review of Cytology. Vol 4. ; 1955:79-102. doi:10.1016/S0074-7696(08)60455-6.

21. Randall $B$, van Amerongen L. Commercial laboratory practice evaluation of air-dried/rehydrated cervicovaginal smears vs. traditionally-fixed smears. Diagn Cytopathol. 1997;16(2):174-176. doi:10.1002/(SICI)10970339(199702) 16:2<174::AID-DC16>3.0.CO;2-J.

22. Safneck JR, Kutryk E, Chrobak A, Harper R, Ravinsky E. Fixation Techniques for Fine Needle Aspiration Biopsy Smears Prepared Off Site. Acta Cytol. 2001;45(3):365-371. doi:10.1159/000327632. 
Table 2. Percentage of Preparation Based on Fixation Method

\begin{tabular}{|c|c|c|c|c|c|c|c|c|}
\hline & \multicolumn{6}{|c|}{ Category } & \multirow{2}{*}{\multicolumn{2}{|c|}{ Total }} \\
\hline & \multicolumn{2}{|c|}{ Not good } & \multicolumn{2}{|c|}{ Well } & \multicolumn{2}{|c|}{ Very good } & & \\
\hline & $\mathrm{n}$ & $\%$ & $\mathrm{n}$ & $\%$ & $\mathrm{n}$ & $\%$ & $n$ & $\%$ \\
\hline Control & 0 & 0 & 4 & 33.3 & 8 & 66.7 & 12 & $100 \%$ \\
\hline Dry Fixation & 4 & 33.3 & 5 & 41.7 & 3 & 25.0 & 12 & $100 \%$ \\
\hline Wet Fixation & 0 & 0 & 8 & 66.7 & 4 & 33.3 & 12 & $100 \%$ \\
\hline
\end{tabular}
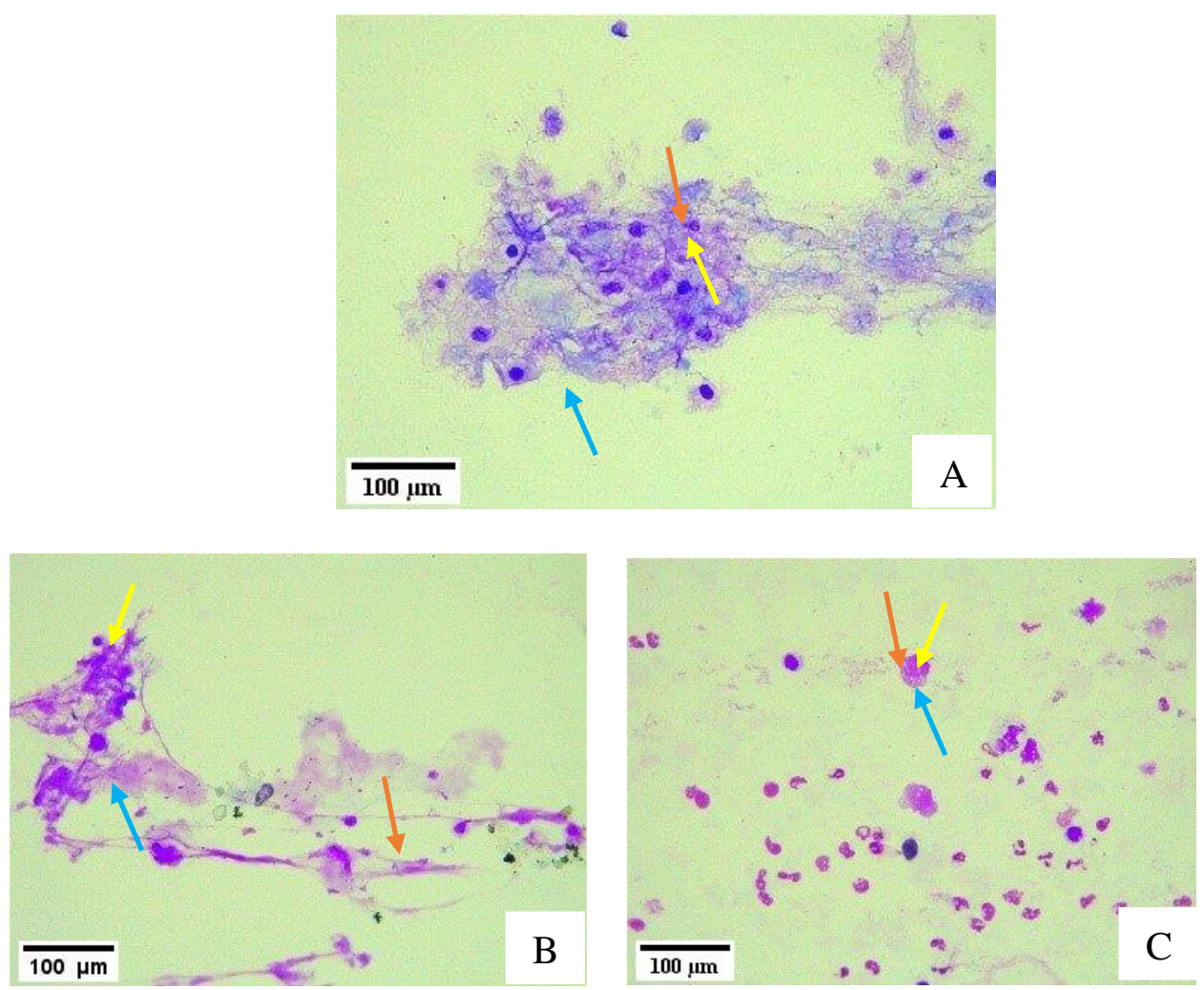

Figure 1. Result for a Well Good Staining Criteria
(A) Dry Fixation
(B) Wet Fixation
(C) Control

Cell form $(\longrightarrow$; Nucleous $(\longrightarrow)$ : Nucleoulous $(\longrightarrow$;

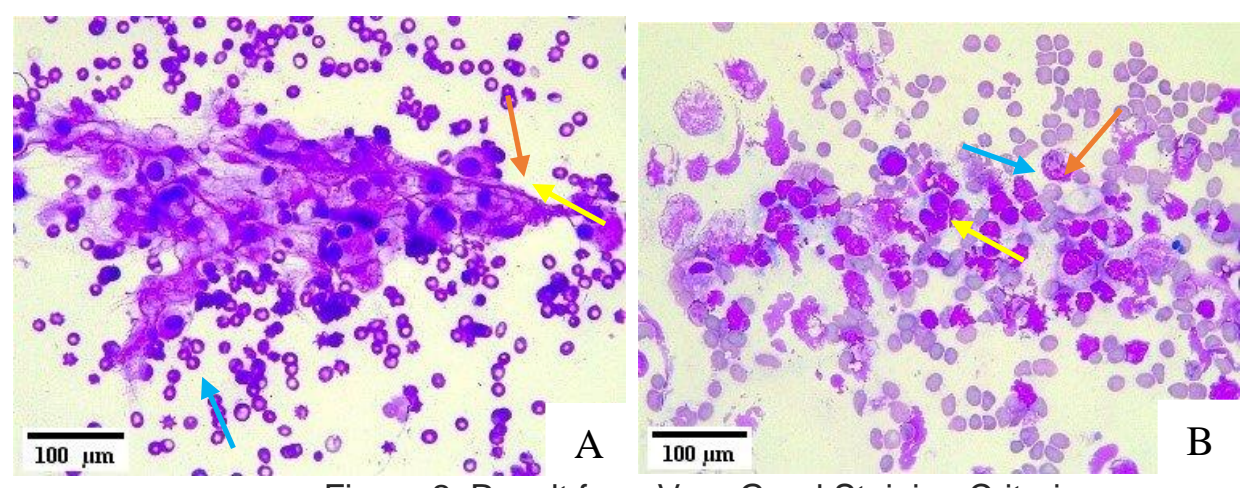
(A) Control
(B) Wet Fixation

Cell form $(\longrightarrow)$; Nucleous $(\longrightarrow$ : Nucleoulous $(\longrightarrow$; 